\title{
Psoriasiform syphilis: A challenge
}

\section{Antônio Pedro Schettini, ${ }^{1}$ José Carlos Sardinha, ${ }^{1}$ Elyana Almeida Marques, ${ }^{1}$ Cesare Massone ${ }^{2}$}

${ }^{1}$ Fundação Alfredo da Matta, Manaus (Amazonas), Brazil; ' ${ }^{2}$ Dermatology Unit, Galliera Hospital, Genoa, Italy

\begin{abstract}
Syphilis is one of the greatest masqueraders in dermatology, like mycosis fungoides or sarcoidosis. In fact, secondary syphilis can simulate different dermatological conditions, not only clinically but also histopathologically. A 34-year-old Brazilian man from Manaus (Amazonas) attended our Department complaining for 2 weeks of asthenia, weight loss and multiple asymptomatic disseminated erythematous papules and scaly plaques with well-defined borders and a tendency to confluence were observed on the trunk, neck and extremities, clinically similar to psoriasis guttata. The skin biopsy and laboratory tests disclosed secondary syphilis and the patient was treated with intramuscular benzathine penicillin with prednisolone, with a complete resolu-
\end{abstract} tion.

Psoriasiform syphilis, which imitates psoriasis, was well known by old dermatologists and was called syphilide psoriasiforme or psoriasis syphilitique. Psoriasiform syphilis is one of the atypical presentations of secondary syphilis and dermatologists should be aware of psoriasiform syphilis in order not to improperly treat syphilis patients with immunosuppressive drugs; also, pathologists should be aware of secondary syphilis histopathological features.

\section{Introduction}

Syphilis is one of the greatest masqueraders in dermatology, like mycosis fungoides or sarcoidosis. In fact, secondary syphilis can simulate different dermatological conditions, not only clinically but also histopathologically. The secondary stage of syphilis corresponds to a lymphatic and hematogenous spread of Treponema pallidum (T.pallidum) with mucocutaneous and systemic manifestations..$^{1,2}$

Fever, malaise, sore throat, localized or generalized lymphadenopathy, hepatosplenomegaly, weight loss, myalgia, arthralgia and headaches are possible prodromal symptoms, simulating different con- ditions such as nodal lymphoma or rheumatological conditions. Skin manifestations are usually classified in early (roseola-like eruption on the trunk), late (generalized maculopapular or papulosquamous polymorphic eruption in successive waves) and specific localized lesions on the palms and soles (symmetric papules and plaques with collarette of Biet), codylomata lata, corona veneris along the hairline, hypopigmented macules on the neck and pachy alopecia. Mucosal involvement occurs in 30\% of cases with perlèche, split papules, mucous patches and a sore throat. ${ }^{1,2}$

Furthermore, atypical manifestations have been described and include nodular, annular, framboesiform and pustular variants, consequent with several differential diagnoses. Nodulo-ulcerative, secondary syphilis (lue maligna) occurs mainly in HIV-positive patients. ${ }^{3}$

In some patients, secondary syphilis may even closely resemble psoriasis, that represents the most frequent inflammatory skin conditions. ${ }^{4,5}$ Therefore syphilis must not be overlooked and before starting a specific systemic treatment for psoriasis, an accurate skin examination and laboratory investigations are mandatory if syphilis is suspected.

\section{Case Report}

A 34-year-old Brazilian man from Manaus (Amazonas) attended our department complaining for 2 weeks of disseminated skin lesions (Figure 1), asthenia and weight loss. His past medical history was unremarkable, he was not taking any drugs and Psoriasis was not recorded in his family history. A physical examination disclosed enlarged retroauricular, inguinal and supratrochlear lymph nodes. At the dermatological examination, multiple asymptomatic disseminated erythematous papules and scaly plaques with well-defined borders, with a tendency to confluence were observed on the trunk, neck and extremities; a few macules with peripheral scaling were also present. The palms presented erythematous-squamous psoriasiform lesions, the soles were not involved, scales were present on the scalp and oral and genital mucosa were normal, as well as nails. He did not report arthralgias.

A skin biopsy performed on a lesion of the trunk to confirm the presumptive clinical diagnosis of guttate psoriasis, showed epidermal hyperplasia (slight irregular acanthosis with elongated rete ridges) with stratum granulosum and moderate parakeratosis, without neutrophils in stratum
Correspondence: Antonio Schettini, Fundação Alfredo da Matta, Rua Umberto Lobato no.16 quadra 36, Planalto, Manaus (Amazonas), Brazil.

Tel.: +55.92984375555.

E-mail: apmschettini@gmail.com

Key words: Syphilis, Psoriasis, Psoriasiform syphilis, Syphilide psoriasiforme.

Contributions: APS: conception and design, analysis and interpretation of data, final approval; JCS, analysis and interpretation of data, drafting, final approval; EAM, analysis and interpretation of data, drafting, final approval; $\mathrm{CM}$, conception and design, analysis and interpretation of data, critical revision, final approval.

Conflict of interest: The authors declare no potential conflict of interest.

Funding: None

Ethical approval: Not applicable.

Consent for publication: Consent was obtained.

Availability of data and material: Data and materials are available by the authors.

Please cite this article as: Schettini AP, Sardinha JC, Marques EA, Massone C. Psoriasiform syphilis: A challenge. Dermatol Rep 2021;13:8830.

Received for publication: 7 August 2020.

Revision received: 8 February 2021.

Accepted for publication: 11 July 2021.

This work is licensed under a Creative Commons Attribution-NonCommercial 4.0 International License (CC BY-NC 4.0).

C. Copyright: the Author(s), 2021

Licensee PAGEPress, Italy

Dermatology Reports 2021; 13:8830

doi:10.4081/dr.2021.8830

corneum. In the dermis a superficial and deep perivascular periadnexal interstitial infiltrate and lymphohistiocytic infiltrate with plasma cells was present and endothelial swelling was observed (Figure 2).

Laboratory investigations disclosed a positive rapid diagnostic test for syphilis, ${ }^{6}$ positive VDRL (titre 1:64) and FTA-ABS tests. HIV and hepatitis $\mathrm{C}$ virus screening were negative.

The diagnosis of psoriasiform secondary syphilis was made. The patient was treated with 2400 UI intramuscular benzathine penicillin with $60 \mathrm{mg}$ prednisolone pre-treatment to prevent a JarischHerxheimer reaction. The lesions healed in 
five weeks.

\section{Discussion and Conclusions}

According to the latest Epidemiological Report, published in October 2019, Brazil identified a total of 158.051 cases of acquired syphilis, with the Amazon region accounting for 3325 cases, and a 59.3\% increase of cases in Amazonas compared to the previous year, much greater than the national increase, which was $28.7 \%$. Considering that sub-notification is a reality in isolated states such as the Amazonas, we can speculate on the real extension of this ancient and current problem.

Reports of atypical forms of syphilis have become more frequent, but the secondary stage is the one that presents the most polymorphism of lesions. This stage, marked by the systemic dissemination of spirochetes and typical skin implication, is undoubtedly the most clinically challenging. ${ }^{2-4}$ One of the atypical forms is the psoriasiform syphilis, which imitates psoriasis (the most frequent inflammatory disease). ${ }^{8}$

This type of presentation was well known by old dermatologists and was called syphilide psoriasiforme or psoriasis syphilitique; ${ }^{9}$ recently Martins et al carefully reviewed old corresponding literature which is mainly reported on old dermatology textbooks. ${ }^{9}$ In modern literature, psoriasiform syphilis has been more frequently reported with presentation mimicking palmoplantar psoriasis, either in patients coinfected with $\mathrm{HIV},{ }^{8-10}$ or in HIV-negative patients. ${ }^{11-15}$ In most cases, syphilis was not correctly diagnosed at the beginning and the patients were treated for psoriasis. ${ }^{11,12,16} \mathrm{To}$ our knowledge, there is not a clear cut explanation of the pathophysiological mechanism of this type of presentation. ${ }^{3} \mathrm{~A}$ familiar history of psoriasis was also not reported by our patient.

Interestingly, our patients papulosquamous lesions involved mainly the trunk and extremities, while the palms presented only few erythematous-squamous psoriasiform lesions and the soles were not involved. Morphology and distribution of the lesions, together with the absence of mucosal involvement in our patient simulated clinical guttate psoriasis, a diagnosis that would have fit very well with the recent history of an exanthematic appearance. The skin biopsy did not show the typical psoriasiform hyperplasia, but a slight irregular acanthosis with elongated rete ridges and the presence of a consistent lymphoid infiltrate with numerous plasma-cells together with endothelial swelling, which were suspicious for syphilis and drove us to specific laboratory investigations.

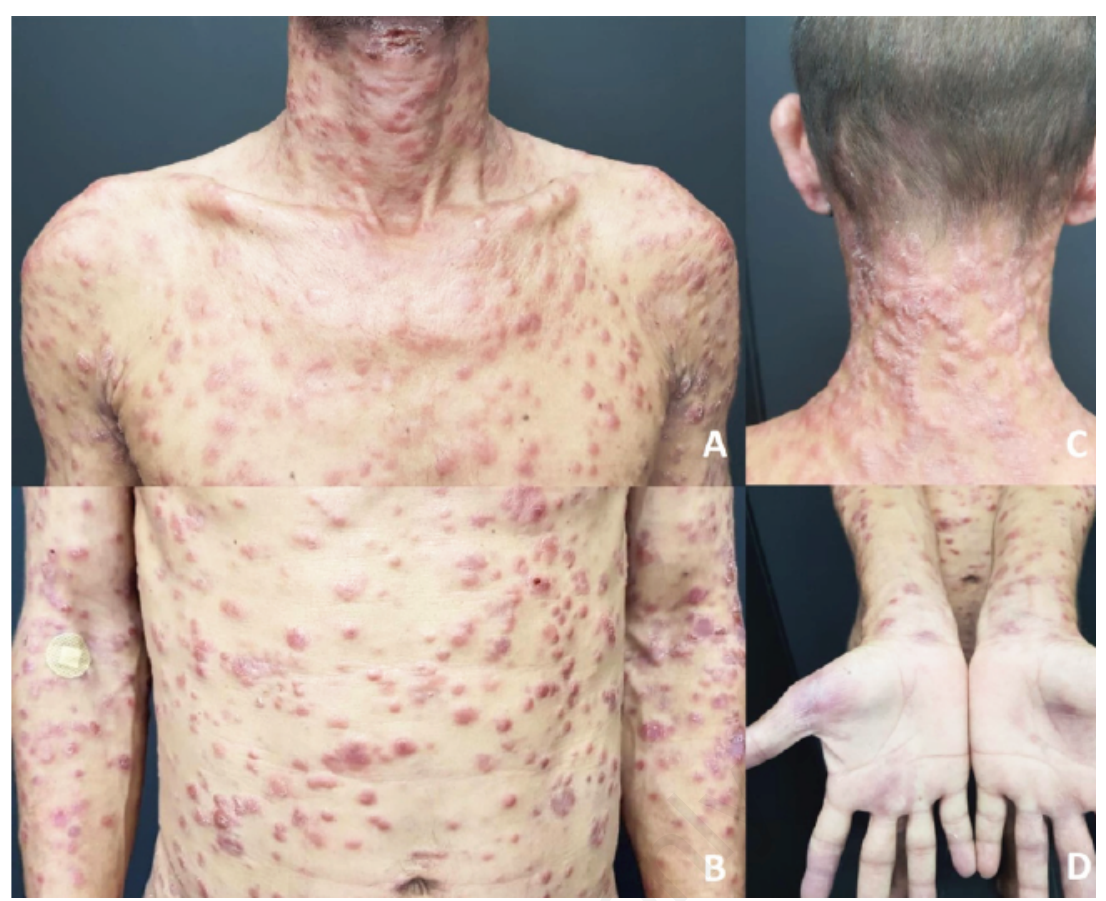

Figure 1. A-D) Multiple asymptomatic disseminated erythematous papules and scaly plaques with well-defined borders, with a tendency to confluence were observed on the trunk, neck and extremities.

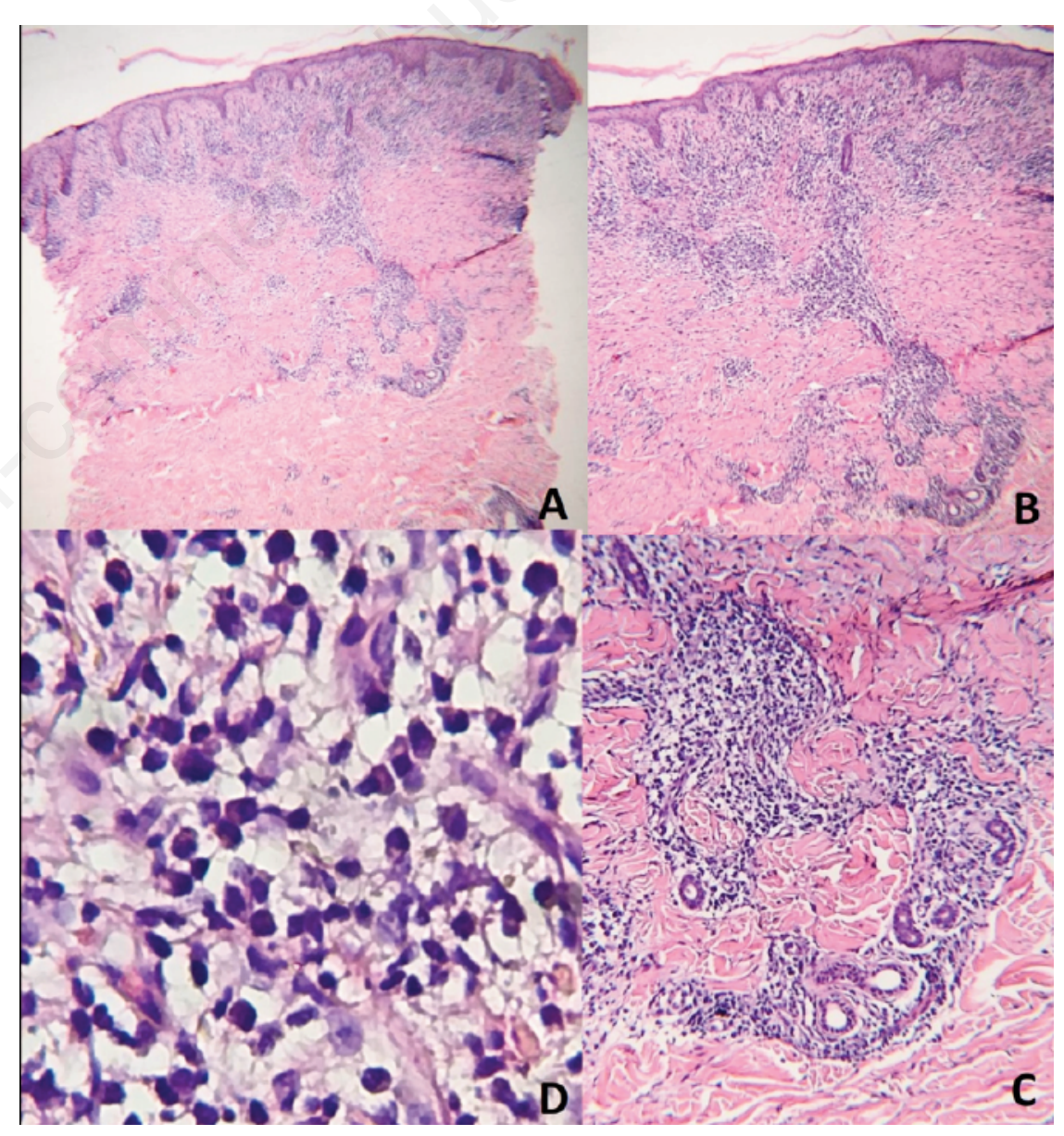

Figure 2. A-C) Slight epidermal hyperplasia, superficial and deep perivascular, periadnexal and interstitial infiltrate $(\mathrm{H} \& \mathrm{E} ; 20 \times, 40 \times$ and $100 \times$, respectively) $\mathrm{D})$ Lymphohistiocytic infiltrate with numerous plasma cells (H\&E; 400x). 
Diagnosis of syphilis is mainly clinical, then supported by laboratory tests; skin biopsies are performed when a clinical presentation is unusual or other conditions must be ruled out. Particularly, secondary syphilis is called The Great Imitator because it can mimic many other different conditions, both clinically and histopathologically. Pityriasis rosea, psoriasis, drug eruption, lichen planus, pityriasis lichenoides and mycosis fungoides are only a few examples, of the many clinical differential diagnosis of secondary syphilis. ${ }^{2-4}$ In our geographical region (Amazonas) endemic diseases like Leishmaniasis and Hansen's Disease must also be considered in the differential diagnosis of secondary syphilis. ${ }^{2-4}$

Histopathology is even more intriguing because the histological features vary according to the stage of the disease, but it is essential to identify potential secondary syphilis patients with ambiguous clinical manifestation through pathology. ${ }^{17,18}$

Alessi et al already described in 1983 the different pathological presentation of secondary syphilis according to the clinical stage, from early macular lesions to late papular ones. The degree infiltrate density and its distribution increases from early (superficial and perivascular) to late stages (superficial, bandlike, and deep perivascular dermatitis); the same for epidermal changes with increasing expression of parakeratosis, spongiosis and exocytosis. ${ }^{19}$

Ackermann wrote that a psoriasiform lichenoid pattern, in which plasma cells predominate is a clue to secondary syphilis, therefore plasma cells are historically considered a significant feature of syphilis. ${ }^{20}$ Contrarily, later Flamm et al. reported that plasma cells can be seen in both syphilis and pityriasis lichenoides and, therefore plasma cells are not a helpful distinguishing feature. ${ }^{9}$ Interestingly, plasma cells can also be absent in about $30 \%$ of syphilis biopsies. ${ }^{18}$

The same authors also reported that the combination of interstitial inflammation, endothelial swelling, irregular acanthosis, and elongated rete ridges should increase the possibility of syphilis, even when no clinical suspicion exists. ${ }^{17}$ Recently Liu \& Li expanded the spectrum of histological features of secondary syphilis, reporting that also necrotic keratinocytes are one of the characteristics of secondary syphilis and is mainly the combination of plasma cells, irregular acanthosis, elongated rete ridges, and endothelial swelling that should increase the likelihood of syphilis. ${ }^{21}$

The main histopathological differential diagnoses are pityriasis lichenoides, pityriasis rosea, and mycosis fungoides, with pityriasis lichenoides being the most difficult one. Flamm et al. reported that there was no unique histopathological feature strongly associated with syphilis. According to these authors, elongated rete ridges and interstitial inflammation favor syphilis over pityriasis lichenoides but there is no definitive feature that can differentiate between them. Secondary syphilis and pityriasis rosea can be distinguished by neutrophils in the stratum corneum, plasma cells, interface dermatitis with lymphocytes, vacuoles, and lymphocytes with ample cytoplasm, all features that are strongly associated with syphilis. ${ }^{17,18}$ Syphilis and mycosis fungoides have a very similar histopathological appearance, but plasma cells and lymphocytes with ample cytoplasm are rare in early mycosis fungoides and can be used as distinguishing features. ${ }^{17,18}$

However, Hoang et al reported that immunohistochemistry (IHC) with a monoclonal antibody to Treponema pallidum demonstrated a sensitivity of $71 \%$, which was superior to the $41 \%$ sensitivity of the silver stain. The presence of T.pallidum was detected mostly at the dermal-epidermal junction, which is probably related to the interaction between the outer membrane protein of T.pallidum and the extracellular matrix. ${ }^{12}$ Although serologic studies remain the gold standard, IHC is a very useful and rapid tool for detecting $T$. pallidum in biopsies of secondary syphilis. ${ }^{22}$

Concerning our case, there was a discrepancy between the clinical picture (thick plaques were prevalent) and the degree of epidermal hyperplasia. However, the presence of interstitial infiltrate with plasma cells, slight irregular acanthosis with elongated rete ridges and endothelial swelling were suspicious for syphilis, then confirmed by serological tests.

Clinicians should be aware of psoriasiform syphilis, in order not to improperly treat a syphilis patient with immunosuppressive drugs, ${ }^{23}$ also pathologists should be aware of the histopathological features of secondary syphilis.

\section{References}

1. Stary G, Stary A. Sexually Transmitted Infections. In: Dermatology 4th Edition. Bolognia JL, Schaffer AV, Cerroni L. Elsevier, 2018

2. Domantay-Apostol GP, Handog EB, Gabriel MT. Syphilis: the international challenge of the great imitator. Dermatol Clin 2008;26:191.

3. Balagula Y, Mattei PL, Wisco OJ, et al. The great imitator revisited: the spec- trum of atypical cutaneous manifestations of secondary syphilis. Int J Dermatol 2014;53:1434-41.

4. Dourmishev LA, Dourmishev AL. Syphilis: uncommon presentations in adults. Clin Dermatol 2005;23:555-64.

5. Trayes KP, Savage K, Studdiford JS. Annular Lesions: Diagnosis and Treatment. Am Fam Physician 2018;98:283-91.

6. Mabey D. Prospective, multi-centre clinic-based evaluation of four rapid diagnostic tests for syphilis. Sex Transm Infect 2006;82:v136.

7. Boletim epidemiológico Secretaria de Vigilancia em Saúde. Numero especial. Out 2019. Biblioteca Virtual em Saúde do Ministério da Saúde. Available from: www.saude.gov.br/bvs

8. Periti I, Zendri E, Ficarelli E, Masotti A, De Panfilis G. Psoriasiform eruption and pharyngitis. Arch Dermatol 2008;144:255-60.

9. Martins CJ, Lima RB, Eyer-Silva WA, et al. Secondary syphilis presenting as Syphilide psoriasiforme: lessons from the older syphilology literature. Rev Inst Med Trop Sao Paulo. 2020;62:e21.

10. Bittencourt Mde J, Brito AC, Nascimento BA, et al. A case of secondary syphilis mimicking palmoplantar psoriasis in HIV infected patient. An Bras Dermatol 2015;90:216-9.

11. Gianfaldoni S, Tchernev G, Wollina U, et al. secondary syphilis presenting as palmoplantar psoriasis. Open Access Maced J Med Sci 2017;5:445-7.

12. Solak B, Kara RO, Erdem T. Palmoplantar syphilis misdiagnosed and treated as palmoplantar psoriasis for 2 years. J Family Med Prim Care 2016;5:506.

13. High WA, Hoang MP, Bergstresser PR. Psoriasis guttata with palmoplantar involvement clinically mimicking secondary syphilis. Cutis 2005;76:358-60.

14. Freitas DMM, Azevedo A, Pinheiro G, Ribeiro R. Psoriasiform papules, condyloma lata, lung nodules and hepatitis: the enormous variability of secondary syphilis manifestations. BMJ Case Rep 2017;2017.

15. Arif T, Adil M, Amin SS, Saeed N. Psoriasiform lesions of glans and palms: a rare presentation in secondary syphilis. Indian J Sex Transm Dis AIDS 2019;40:70-2.

16. Peng RR, Shang S, Chen J, Shi M, Long FQ. Delayed Onset and Protracted Course of Psoriasis-like Secondary Syphilitic Lesions in a HIV-sero-positive Man Who has Sex with Men: A Case Report. Acta Derm Venereol 2019;99:1197-8. 
17. Flamm A, Parikh K, Xie Q, et al. Histologic features of secondary syphilis: A multicenter retrospective review. J Am Acad Dermatol 2015;73:1025-30.

18. Flamm A, Alcocer VM, Kazlouskaya V, et al. Histopathologic features distinguishing secondary syphilis from its mimickers. J Am Acad Dermatol 2020;82:156-60.
19. Alessi E, Innocenti M, Ragusa G. Secondary syphilis. Clinical morphology and histopathology. Am J Dermatopathol 1983;5:11-7.

20. Ackermann AB, Guo Y, Vitale P. Clue 62. In: Clues to diagnosis in dermatopathology. ASCP Press, Chicago; 1992: 246-7.

21. Liu XK, Li J. Histologic Features of Secondary Syphilis. Dermatology
2020;236:145-50.

22. Hoang MP, High WA, Molberg KH. Secondary syphilis: a histologic and immunohistochemical evaluation. J Cutan Pathol 2004;31:595-9.

23. Cather JC, Cather JC, Menter MA. Psoriasiform lesions on trunk and palms. Proc (Bayl Univ Med Cent) 2003;16:236-38. 